\title{
Delamanid expanded access novel treatment of drug resistant tuberculosis
}

\author{
This article was published in the following Dove Press journal: \\ Infection and Drug Resistance \\ 29 October 2015 \\ Number of times this article has been viewed
}

\author{
Roxana Rustomjee' \\ Alimuddin Zumla ${ }^{2,3}$ \\ 'South African Medical Research \\ Council, Cape Town, South Africa; \\ ${ }^{2}$ Division of Infection and Immunity, \\ University College London, London, \\ UK; ${ }^{3}$ NIHR Biomedical Research \\ Centre, University College Hospitals \\ NHS Foundation Trust, London, UK
}

\begin{abstract}
Tuberculosis (TB) remains a global emergency and is one of the most common infectious disease causes of death in developing countries. Current treatment regimens for multi-drug resistant TB are associated with low treatment success rates, are toxic, and require long duration of treatment. The need for shorter and more effective treatment regimens is urgent. Delamanid (Deltyba, or formerly known as OPC-67683) is a new dihydro-imidazooxazole anti-TB drug active against resistant forms of pulmonary TB. Delamanid kills Mycobacterium tuberculosis by inhibiting the synthesis of mycolic acids required for cell wall synthesis. Whilst delamanid has been included in the WHO Model List of Essential Medicine by the World Health Organization Expert Committee on Selection and Use of Essential Medicines and in international guidance for the treatment of multi-drug resistant TB since April 2014, its access in countries with the greatest need, has proven challenging. This review provides an update on currently available clinical safety and efficacy data on delamanid and offers a discussion on research priorities and recommendations for expedited, expanded access.
\end{abstract}

Keywords: delamanid, tuberculosis, drug resistance, MDR-TB, expanded access

\section{Introduction and background}

Current treatment regimens for multi-drug resistant tuberculosis (MDR-TB) are associated with low treatment success rates, are toxic, and require long duration of treatment. ${ }^{1,2}$ The need for shorter and more effective treatment regimens is urgent. ${ }^{3}$ Delamanid (Deltyba ${ }^{\mathrm{TM}}$, or formerly known as OPC-67683) is a new nitro-dihydroimidazooxazole, anti-TB drug which now significantly broadens the repertoire of treatment options particularly for adults with resistant forms of pulmonary TB. ${ }^{4}$ It has been included in the World Health Organization (WHO) Model List of Essential Medicine by the WHO Expert Committee on Selection and Use of Essential Medicines. ${ }^{5}$ While this drug has been included in international guidance for the treatment of MDRTB since April 2014, ${ }^{6}$ access, particularly in countries with the greatest need, has proven challenging. By end of December 2014, less than ten patients outside clinical settings had received delamanid. ${ }^{5}$ Recently, at the WHO Global Laboratory Initiative Partners Forum in Geneva; heralded as the "FighTBack Initiative" Otsuka (the drug's developer) announced:

A targeted access donation program, innovative research and development of new products for MDR-TB, optimized patient management, and collaborative capacity building, including working with communities using new approaches to ensure delamanid is administered safely and responsibly to minimize the threat of drug-resistance. ${ }^{7}$
Correspondence: Roxana Rustomjee South African Medical Research Council, Francie van Zijl Drive, Parow Valley, 7505 , Cape Town, South Africa Tel +27 722723009

Email roxana.rustomjee@mrc.ac.za 
It is anticipated that in upcoming months an action plan developed with strategic partners will attempt to reach about half of the 27 highest-burden MDR-TB countries. ${ }^{7}$

Whilst this long awaited donation is aimed at delivering, at least, in part, on the " 20 by 2020 " goal, which is to ensure delamanid reaches $20 \%$ of all MDR-TB diagnosed and treated patients by $2020 ; 8$ to date limited information can be obtained as to the timeframe and mechanism for this expansion of access. ${ }^{7,8}$ Further, there are significant exclusions to the countries listed in the donation announcement, eg, South Africa, with amongst the highest burden of MDR-TB and amongst the largest patient contributors to the trials that produced the evidence for the safety and efficacy of delamanid, is precluded from the donation. ${ }^{7}$ It is ethically imperative that delamanid becomes available in countries where clinical trials have been performed. ${ }^{9}$ Thus far, only a few of those in dire need ( $<100$ patients), have successfully accessed this drug through either compassionate use or post-trial obligations of the trial sponsors to patients and their communities. ${ }^{9}$ Most importantly, medicine donation programs, while offering temporary relief to defined problems, are neither a long-term solution to underfunded health systems nor a solution to the lack of access to medicines in poor countries especially for diseases that are endemic, require long or lifelong treatment or large numbers of treatments. ${ }^{10,11}$ Additional risks of limited drug donation schemes preclude long-term planning, sustainability, and incentivize donor companies to benefit from tax incentives. ${ }^{11}$ The "Drugs For Neglected Diseases Working Group", of the Médecins Sans Frontières, compared the donation model with other models that could improve access to essential medicines, including the purchase of generics, concessionary pricing, discounted pricing, and differential pricing; and demonstrated that drug donations may cost the public sector of a donor country more than four times as much as other models that achieve the same end result. ${ }^{11}$ Therefore until this plan, as well as specially designed, country specific, targeted expanded access plans are realized, a significant proportion of the estimated 500,000 people ${ }^{1}$ who develop MDR-TB, of which about $10 \%{ }^{1}$ have extensively drug-resistant (XDR)-TB, will fail to access optimal treatment options. Of those who do, alarmingly, only half of patients on treatment, will achieve treatment success - less than $20 \%$ for XDR-TB (the most untreatable and lethal form of TB). ${ }^{2,3,12}$ Addressing the diagnostic challenges, dismal access and highly limited and frequently toxic treatment options for MDR-TB patients remain a priority. As yet, there has been limited progress in rapidly deploying the two new and recently available drugs. ${ }^{5}$ This urgent need to improve treatment outcomes for patients with a life-threatening condition and reduce MDR- and XDR-TB transmission by achieving early culture conversion stands in equipoise with safety stipulations for full registration and global access of new drugs. This review provides an update on currently available clinical safety and efficacy data on delamanid and offers a discussion on research priorities and recommendations for expedited, expanded access.

\section{Guidance on use}

Global public health organizations ${ }^{5-8}$ and advocacy groups, ${ }^{9}$ driven by a public health imperative to strengthen the rationale for rapid access of delamanid, as well as bedaquiline, the only other new TB drug in late clinical development, ${ }^{13}$ hastened to provide an evaluation of the evidence for safety and efficacy claims ahead of a definitive Phase III trial. ${ }^{14}$ The WHO's Interim Policy Guidance on the use of delamanid for the treatment of MDR-TB (2014), ${ }^{6}$ while fully understanding the limitations of both the data presented and the conclusion derived thereof, nonetheless, made an interim policy recommendation for the restricted use of delamanid. This WHO conditional recommendation for delamanid's use is similar but considerably broader to that for bedaquiline; ${ }^{5}$ recommending its use when an effective treatment regimen cannot be designed due to resistance or intolerance to existing drugs. ${ }^{6}$ However, it goes further by recommending delamanid use in patients with high risk of poor outcomes (ie, extensive or advanced disease). By implication delamanid is potentially suitable for a larger number of patients. ${ }^{6}$ Until further evidence ensues, patients with QTc $>500 \mathrm{~ms}$ are restricted from delamanid use and the two drugs (delamanid and bedaquiline) may not be jointly administered. ${ }^{6}$ Nevertheless, the potential number of patients likely to benefit from delamanid treatment would all be XDR-TB, pre-XDR-TB and up to $10 \%$ of MDR-TB patients. With due consideration for currently available data on the limited treatment strategies for these patients, with limited clinical evidence to support the use of existing drugs and with a significant number of these drugs being used "offlabel", it is important to ensure that the use of delamanid is considered and that the broader recommendations for its use are known. ${ }^{2-6}$ These recommendations came close on the heels of the European Public Assessment Report ${ }^{15}$ and conditional regulatory approval by the European Medicines Agency (EMA) ${ }^{16}$ and Japanese regulatory authority ${ }^{17}$ in April and July 2014 respectively; becoming only the second new drug from a new drug class (after bedaquiline) to receive such approval in over 40 years. ${ }^{16,17}$ 
This proactive decision by Stringent Regulatory authorities, apart from setting a new path of expedited/accelerated approval of much needed life-threatening anti-TB medicines, simultaneously demanded intensive pharmacovigilance efforts and a fixed time-frame of 2 years to provide data in support of the safety and efficacy claims. ${ }^{15}$ Full (unconditional) registration will depend on the further evaluation of safety and efficacy addressing the concerns with the data and quality of evidence. Apart from the results of a fully enrolled Phase III trial expected mid-2017, ${ }^{14}$ two additional trials of pediatric safety and efficacy and optimal dosing are expected in $2017^{18}$ and $2018 .{ }^{19}$

According to the Committee for Medicinal Products for Human Use, the conditional marketing authorization was granted:

[...] to a medicinal product that fulfils an unmet medical need when the benefit to public health of immediate availability outweighs the risk inherent in the fact that additional data are still required. The marketing authorization holder is likely to provide comprehensive clinical data at a later stage. ${ }^{20}$

The EMA recommendations, ${ }^{15,16,20}$ together with the WHO Policy recommendation, ${ }^{6}$ and the Clinical Commissioning Policy: Bedaquiline and Delamanid for MDR- and XDR-TB, prepared by NHS England Specialised Services Clinical Reference Groups for Infectious Diseases and Respiratory Medicine $^{21}$ in response to the EMA market authorization provide detailed summaries of this evidence; additional conditions of use including prescribing restrictions are elucidated. ${ }^{15,16,20,21}$ Despite these efforts few additional patients have received delamanid ${ }^{5}$ and most significantly, plans for access to delamanid outside of clinical trials in high burden, less developed countries remained elusive in the past year. ${ }^{5}$

\section{Mechanism of action; indication}

Delamanid, a nitro-dihydro-imidazooxazole, is an orally dosed prodrug whose primary metabolites likely kill tuberculous mycobacteria by blocking the synthesis of mycolic acids (ie, stopping the bacteria from creating building blocks important for their cell walls). ${ }^{4}$ Nitroimidazoles also appear to kill the TB bacteria by poisoning them with nitric oxide, which the drugs release when metabolized. ${ }^{4}$ Theoretically this presents novel and complementary mechanisms of action to currently used optimized background regimens. ${ }^{2}$ Therefore, according to its registered indication, this designated, "orphan drug" may be used to treat "pulmonary MDR-TB (MDRTB of the lungs) in adults for whom an effective treatment regimen cannot otherwise be constructed due to resistance or tolerability".22

\section{Evidence for the efficacy of delamanid in the treatment of MDR-TB}

Published and/or presented clinical studies of delamanid are few, ${ }^{23-28}$ and comprise: i) drug-drug interaction studies with anti-retroviral drugs, ${ }^{23,24}$ ii) an early bactericidal activity Phase IIa dose-ranging trial of delamanid monotherapy in 48 uncomplicated smear positive pulmonary TB over 14 days; ${ }^{25}$ and iii) the pivotal Phase IIb trial 204 of delamanid plus an optimized background regimen in pulmonary MDR-TB patients that assessed culture-conversion over 2 months of therapy. ${ }^{26}$ Safety and efficacy assessments were further evaluated in the 6-month open-label extension trial $208^{27}$ and the 24-month observational follow-up of these patients. ${ }^{28}$ The majority of early studies are unpublished but contributed to the EMA authorization report. ${ }^{16}$ Preclinical evidence as well as published and unpublished clinical studies are evaluated by Lewis and Sloan. ${ }^{29}$

\section{Short-term efficacy}

Defined as a patient achieving a series of at least five consecutive weekly cultures negative for growth of Mycobacterium tuberculosis (without subsequent positive cultures) served as the primary efficacy analysis. ${ }^{26}$ Trial 204 was conducted at 17 sites in 9 countries. ${ }^{26}$ The promising results of a $16 \%$ increment in the 2-month culture conversion rates in the delamanid versus placebo arms of the 481 patient double-blind, placebo-controlled pivotal Phase II trial (45.4\% vs $29.6 \%$, $P=0.008$ ), as well as the significantly reduced time to culture conversion in the secondary endpoint led the evidence for the short-term efficacy and safety of delamanid, and founded the basis of the EMA approval. ${ }^{15,16}$

\section{The longer term efficacy}

Accrued from the follow-up of individuals enrolled into a 6-month, non-randomized extension study of trial 204, ${ }^{27}$ where there was a choice to treat, as determined by the attending clinician, with delamanid, at either $100 \mathrm{mg}$ or $200 \mathrm{mg} .{ }^{25} \mathrm{At}$ a highly variable interval (as long as 4 months) post-trial 204, participants received delamanid for 6 months (placebo arm of trial 204), or 8 months with a break in the middle, and those who did not participate in the 6-month study received delamanid for either 2 months or not at all. In addition to the data presented by the manufacturer, the EMA re-examined the 2-year efficacy (and safety) profile. ${ }^{15,16}$ Despite significant 
limitations in the quality of the data including differing dosing and treatment length and a high rate of cohort attrition, 75\% of those who received delamanid for 6 months or more had no bacteria in their sputum (participants who chose the 6-month study), ${ }^{27}$ compared with $55 \%$ who received delamanid for 2 months or less (participants who did not choose the 6-month study). Mortality was only $1 \%$ of those who received longterm delamanid, versus $8.3 \%$ of those who received 2 months of no delamanid $(P<0.001)$. $^{15,27,28}$

A post hoc analysis of XDR-TB patients in Otsuka's multi-phase clinical trial of delamanid (Trial 204/208/116) showed that patients receiving delamanid for 2 months had a higher rate of 2-month sputum-culture conversion than patients receiving placebo (7 of 16 [44\%] vs 1 of 10 [10\%], $P=0.10) .{ }^{30}$ There was also a trend towards lower mortality among patients treated with delamanid for 6 months or more when compared to patients treated with delamanid for 2 months or less ( 0 of 17 vs 2 of 9 [22\%], $P=0.11$ ). ${ }^{27}$ There were also trends towards higher rates of sustained sputumculture conversion (13 of 17 [77\%] vs 4 of 9 [44\%], $P=0.19$ ), and rates of successful treatment outcomes (11 of 17 [65\%] vs 4 of 9 [44\%], $P=0.42$ ) among patients treated for 6 months compared to those treated for 2 months or less. ${ }^{30}$

\section{Safety}

While it is clear that more data are needed to support the safety of delamanid, early evidence appears reassuring with the most commonly reported and resolvable adverse events being nausea, vomiting, and dizziness (in about one third of people taking it); low serum potassium levels; paresthesia; anxiety, and tremors. ${ }^{15,23,29}$ The risk of a serious adverse event was about the same for patients receiving delamanid or placebo in the 2-month trial, ${ }^{26}$ and was slightly higher in the $200 \mathrm{mg}$ twice daily dosing than the lower $100 \mathrm{mg}$ dose. QT prolongation, the most troubling side effect of delamanid, is also caused by other MDR-TB drugs like bedaquiline, clofazimine, and moxifloxacin. No studies have been done yet to show whether the effects of these drugs on heart rhythm are additive, or if the drugs are safe to use together. A National Institutes of Health AIDS Clinical Trials Group study (A5343) will investigate drug-drug interactions and combined QT effects of co-administered bedaquiline and delamanid, with results expected in $2016 .{ }^{31}$ For patients with extensive drug resistance and limited treatment options, potential benefits of combining two or more of these drugs may outweigh potential risks, particularly when regular monitoring for heart safety (ie, electrocardiograms) is available. The same argument is relevant when faced with treating patients with comorbidities and concomitant medication, including other anti-TB drugs, new TB drugs and particularly anti-retrovirals. This expanded safety information with the inclusion of special populations of children, pregnant women, and the elderly will not be possible within the prescribed confines of the Phase III trial and define the research priorities going forward.

\section{Dosing}

Deltyba is formulated in $50 \mathrm{mg}$ oral tablets. ${ }^{22}$ The recommended dose is $100 \mathrm{mg}$ twice daily, given for 6 months (24 weeks), as part of an MDR-TB treatment regimen. ${ }^{22}$ The metabolic pathway has not been fully elucidated, but serum albumin is thought to be the primary route of metabolism with minimal involvement of hepatic cytochromes. No dose adjustment for mild or moderate renal impairment and mild hepatic impairment is needed but additional data are needed for severe renal impairment and moderate and severe hepatic impairment. ${ }^{22} \mathrm{Co}$-administration with food (especially fatty meals) has been shown to have a significant impact on drug absorption and it is recommended that delamanid be dosed with food. ${ }^{22}$ Hypoalbuminemia has been associated with an increased risk of QTc prolongation. ${ }^{22}$ Therefore, delamanid is contraindicated in patients with albumin $<2.8 \mathrm{~g} / \mathrm{dL}$. Co-administration with strong CYP3A inhibitors (eg, lopinavir/ritonavir) and fluoroquinolones may also lead to QTc prolongation and frequent monitoring of electrocardiograms throughout the treatment period is recommended when co-administration with these drug classes is required. ${ }^{22}$

\section{Select research priorities}

While clinical studies to date indicate an overall good safety and early efficacy profile, the small number of patients $(<1,000)$ who have been exposed to delamanid to date and the inherent inability of current Phase II trial designs to accurately predict true clinical efficacy are important considerations when prescribing delamanid to patients. ${ }^{22-30}$ Patients should be followed closely both during and after treatment with delamanid to assess for potential toxicity and treatment efficacy. There is a pressing need for evidence to support a treatment duration of longer than 6 months, the potential for combined therapy with bedaquiline concurrently (ie, delamanid treatment to follow bedaquiline), and the inclusion in new shorter regimens including in combination with bedaquiline, once the AIDS Clinical Trials Group A5343 Trial results provide support for this as well as further studies on drug interactions, and cardiac adverse effects. Additionally several knowledge gaps merit mention as future research priorities. 
To date, delamanid's use in pediatric populations, albeit a few pediatric cases under compassionate use, ${ }^{32}$ have not been evaluated, and the dosage, safety, and efficacy of delamanid in children and adolescents ( $<18$ years) has not yet been established. Two trials, a pediatric dose ranging study (estimated completion March 2016) ${ }^{18}$ and a 6-month open label safety, efficacy, and pharmacokinetic study (estimated completion April 2017) in children as young as 6 years old are ongoing. ${ }^{19}$ These studies will also provide an important basis for evaluating delamanid in children aged six and younger. ${ }^{18,19}$ Additionally, by consensus, due to the high burden of TB disease and the limited pediatric MDR-TB treatment options, a panel of international experts advocates that children should be included in studies at the early phases of drug development and should be an integral part of the clinical development plan, rather than studied after regulatory approval in adults is obtained. ${ }^{33}$ Future Phase III trials of delamanid should heed this recommendation.

There are very little published clinical data to guide the use of delamanid during the perinatal period. The outcomes of pregnancies that have occurred during clinical trials and clinical use to date have not been published. Delamanid and its metabolites have also been shown to be excreted into breast milk. ${ }^{13}$ One documented case of pregnancy in a 32-year old Asian woman who conceived approximately 34 days after the last dose of delamanid in study $204^{26}$ and study $208^{27}$ resulted in pre-term labor at 33 weeks gestation that that did not progress and at nearly 39 weeks, she delivered a healthy male by cesarean section. ${ }^{15}$ Additional data are urgently needed to better inform the risk:benefit profile of delamanid in this patient population.

Clinical trials of delamanid to date have not included significant numbers of HIV infected individuals, especially those with low CD4 counts and or late stage disease. . $5,23,29^{-1}$ The current Phase III trial underway includes a sub-study of HIV infected individuals and will provide critical information about the use of delamanid in this population. ${ }^{14}$ Additionally, potential drug-drug interactions between delamanid and anti-retroviral treatment (combination antiretroviral therapy) regimens that include ritonavir require further elucidation to guide safe use of this drug. There was a modest increase in exposure to delamanid and its metabolites when co-administered with the protease inhibitor lopinavir/ritonavir in healthy volunteer studies. ${ }^{23}$ This has potential implications for increased toxicity (in particular QTc prolongation). Given this interaction, as well as the high rates of drug resistance and treatment failure associated with HIV infection, ${ }^{12}$ better understanding of the use of delamanid for treatment of drug resistant TB in this population deserves high priority.

Preclinical studies have shown that the in vitro frequency of spontaneous resistance is high. ${ }^{15}$ Mutations in any one of the five genes associated with the mycobacterial co-enzyme (F420) responsible for metabolic activation of delamanid is suggested as the mechanism for resistance against delamanid. ${ }^{15}$ These enzymes and co-factors are likely non-essential thereby allowing multiple viable pathways to resistance formation. ${ }^{15}$ The spontaneous resistant rate for delamanid is similar to that of isoniazid when assessed in a common lab strain of TB (H37RV), therefore it is expected that the emergence of resistance to delamanid will be high unless it is paired with other potent anti-TB drug(s). ${ }^{15}$ Given the potential for spontaneous resistance formation to delamanid, epidemiologic monitoring concurrent to clinical use is an important research priority. ${ }^{15}$ Additionally, the elucidation of molecular markers of resistance and development of rapid diagnostics capitalizing on this information will be critical to ensuring appropriate use of this drug and preventing widespread development of resistance at the population level.

Lastly, delamanid has shown dose dependent bactericidal activity against dormant Mycobacterium bovis bacillus CalmetteGuérin in an in vitro hypoxic system and also has ability to kill intracellular M. tuberculosis. ${ }^{15}$ These characteristics suggest a potential role for this drug in the treatment of latent TB infection, especially in those exposed to MDR- and XDR-TB. Clinical evaluation in this context warrants further consideration.

\section{Licensing strategy}

The Phase III trial completed enrollment in late 2013, with an estimated completion date of May 2016. ${ }^{14}$ Sufficiently compelling evidence from the Phase II trial paved the way for accelerated and conditional registration in Europe and Japan. ${ }^{15-17}$ Now is the time to seek regulatory approval in countries where the drug is most needed. The process of establishing clinical guidelines for the addition of delamanid in patients with MDR- and XDR-TB with proven resistance to fluoroquinolones and/or intolerance to second line anti-TB drugs is already underway and discussions with regulators and ministries of health should be initiated without delay if not already commenced. These discussions should include pricing and procurement; governance; mechanisms of funding and an assessment and capacitating of national TB programs so patients are managed at a recognized MDR-TB center; that treatment with these new drugs will be guided by individual patient assessments and ongoing care will be provided by individuals with expertise in treating MDR-TB patients. 
Centers will follow WHO recommended regimens ${ }^{6}$ and will have access to adequate outreach and laboratory support. Drugs will be administered under closely monitored conditions, adhering to best practices in treatment delivery, to enable optimal drug effectiveness and safety. ${ }^{6}$ The responsibility to ensure delamanid is available as soon as possible rests with both the countries that need the drug as well as with Otsuka (the manufacturing and licensing company). Although there is no quick fix, many measures can be employed to improve access to medicines in developing countries. ${ }^{34}$ Traditionally, less developed countries have increased access to essential drugs by donations or preferential/tiered-pricing strategies that allow for the lower pricing of drugs in certain markets. While such measures retain patent protection, they can also make health economies reliant on products that might not be so affordable in the future (and volumes of medicines needed for diseases like TB and HIV/AIDS may be difficult for a manufacturer to fulfill). ${ }^{34}$ Crucially, "preferential pricing" does not improve, and can even damage, the competitiveness of local markets. ${ }^{34}$ Recent, novel licensing strategies such as voluntary (by the manufacturing company) and compulsory (by the countries) may provide innovative solutions to expanding licensing options particularly for drug companies that have developed drugs in partnerships with public funders. ${ }^{34}$ In the latter, World Trade Organization agreements allow for member countries to compulsorily license medicines in certain circumstances if they believe the health of the nation requires it. ${ }^{34}$ This has happened recently in Indonesia; the government issued compulsory licenses for seven HIV medicines with the owner companies to receive $0.5 \%$ in royalties. In September 2014, US pharmaceutical company Gilead Sciences agreed to license hepatitis $\mathrm{C}$ antivirals sofosbuvir and ledipasvir to seven India-based manufacturers. ${ }^{34}$ This example of voluntary licensing enabled the production and sale of more affordable versions in 91 developing countries. ${ }^{34}$

\section{Global access}

Thus far Deltyba has been launched only in the United Kingdom and Germany despite marketing authorization for the whole of Europe. The cost of a 6-month treatment course in these countries is prohibitive at over US\$33,000 for a 6-month course of $100 \mathrm{mg}$ twice daily. ${ }^{9}$ High TB burden countries in Eastern Europe such as Estonia, Latvia, Lithuania, and Romania, as yet have not had access to the drug outside of enrollment in the clinical trials - specific plans to broaden the access to this drug have not been made public neither are labeling and educational materials available for most countries, which may further delay access where there is an urgent need for access to MDR-TB treatment options. ${ }^{9}$

Further, to date, despite an intention to widen access to delamanid outside the European Union and Japan, high TB burden countries or where clinical trials were conducted such as People's Republic of China, Philippines, Egypt, Korea, Moldova, Peru, and South Africa have not been engaged in terms of developing a licensing strategy. ${ }^{9}$ Nor has expanded access under compassionate use been overwhelmingly enthusiastic. ${ }^{9}$ Compassionate use programs apart from being vital for patients with XDR-TB, pre-XDR-TB, or serious challenges tolerating MDR-TB treatment will provide much needed efficacy and safety information in these difficult to treat sub-populations. The current limited access to this option is approved on a case-by-case basis via an emailed request to the company directed to medical@otsuka.de. There is ample opportunity to broaden the scope and to make this option available through proper country mechanisms.

\section{Conclusion}

At best providing care and treatment to MDR-TB patients is fraught with clinical challenges including progressively evolving or changing resistance patterns, patients with extrapulmonary TB and such as pre-XDR-TB and XDR-TB or any and many combinations of all these scenarios that complicate the evaluation of any new TB drug. Deltyba is an important new drug for the treatment of MDR-TB which has been given accelerated approval for use as a part of a combination regimen to treat pulmonary MDR-TB.

While Deltyba has been theoretically available for clinical use on a limited basis due to conditional approval by the EMA and Japanese regulatory authorities, ${ }^{16,17}$ clinical availability to date has been limited. Recent initiatives by the WHO and Otsuka are a promising development to address this issue and it is critical that promise gives way to active implementation. ${ }^{1-8}$ Additionally, in light of limited clinical data to guide the optimal use of this new drug, expanded access programs and clinical research opportunities should be exploited to provide a larger pool of data to inform both clinical practice and future studies. Key populations currently lacking data include pregnant women, pediatric populations, and those infected with HIV. ${ }^{15,29}$ Concerns about a relatively low threshold to resistance also merit further research to prevent drug resistance from limiting the full potential of this drug in individual and population levels. ${ }^{15}$

\section{Disclosure}

The authors report no conflicts of interest in this work. 


\section{References}

1. World Health Organization. Global tuberculosis report 2014. Geneva: World Health Organization; 2014. Available from: http://www. who.int/tb/publications/global_report/en/. Accessed September 27, 2015.

2. Zumla A, Chakaya J, Centis R, et al. Tuberculosis treatment and management - an update on treatment regimens, trials, new drugs, and adjunct therapies. Lancet Respir Med. 2015;3(3):220-234.

3. Ma Z, Lienhardt C, McIlleron H, Nunn AJ, Wang X. Global tuberculosis drug development pipeline: the need and the reality. Lancet. 2010; 375(9731):2100-2109.

4. Matsumoto M, Hashizume H, Tomishige T, et al. OPC-67683, a nitrodihydro-imidazooxazole derivative with promising action against tuberculosis in vitro and in mice. PLoS Med. 2006;3(11):e466.

5. World Health Organization. The Selection and Use of Essential Medicine Report: 20-24 April, 2015. Geneva: World Health Organization. Available from: http://www.who.int/medicines/publications/essentialmedicines/Executive-Summary_EML-2015_7-May-15.pdf. Accessed June 6, 2015.

6. World Health Organization. The Use of Delamanid in the Treatment of Multidrug-Resistant Tuberculosis Interim Policy Guidance 2014. Geneva: World Health Organization. Available from: http://apps.who. int/iris/bitstream/10665/137334/1/WHO_HTM_TB_2014.23_eng. pdf?ua=1. Accessed September 27, 2015.

7. World Health Organization [homepage on the Internet]. An initiative to extend access to a new TB drug; 2015. World Health Organization. Available from: http://www.who.int/tb/features_archive/otsuka_2015/ en/. Accessed September 27, 2015.

8. UNITAID [homepage on the Internet]. UNITAID joins health partners to launch new treatment for drug-resistant TB; 2015. UNITAID. Available from: http://www.unitaid.eu/en/resources/press-centre/20news/statements/1433-unitaid-joins-health-partners-to-launch-newtreatment-for-drug-resistant-tb. Accessed September 27, 2015.

9. Ahuja SD, Ashkin D, Avendano M, et al. Multidrug resistant pulmonary tuberculosis treatment regimens and patient outcomes: an individual patient data meta-analysis of 9,153 patients. PLoS Med. 2012;9(8): e1001300.

10. World Health Organization. Guidelines for Medicine Donations Revised 2010. Geneva: World Health Organization. Available from: http:// www.who.int/medicines/publications/med_donationsguide2011/en/. Accessed September 27, 2015.

11. Guilloux A, Moon S. Hidden Price Tags: Disease-Specific Drug Donations: Costs and Alternatives. Access to Essential Medicines Campaign, Médecins Sans Frontières, Geneva, Switzerland; 2014. Available from: http://www.researchgate.net/publication/242325532 Hidden_Price_Tags_Disease-Specific_Drug_Donations_Costs_and_ Alternatives. Accessed September 27, 2015.

12. Treatment Action Group [homepage on the Internet]. Treatment Action Group Applauds European Approval of New Drug to Fight Tuberculosis, Demands Expanded Access and Affordable Pricing. Treatment Action Group; 2013. Available from: http://www.treatmentactiongroup. $\mathrm{org} / \mathrm{tb} / \mathrm{press} / 2013 / \mathrm{tag}$-applauds-european-approval-new-drug-fighttuberculosis-demands. Accessed September 27, 2015.

13. World Health Organization. Interim Policy Guidance for the Use of Bedaquiline in the Treatment of MDR-TB. Geneva: World Health Organization; 2013. Available from: http://www.who.int/tb/challenges/ mdr/bedaquiline/en/. Accessed September 27, 2015.

14. Otsuka Pharmaceutical Development and Commercialization, Inc. Safety and Efficacy Trial of Delamanid for 6 Months in Patients With Multidrug Resistant Tuberculosis. Available from: https://clinicaltrials.gov/ ct2/show/NCT01424670?term=phase+III+trial+delamanid\&rank=1. NLM identifier: NCT01424670. Accessed September 27, 2015.

15. European Medicines Agency. European Public Assessment Report: Deltyba (delamanid). 2014 April 28. Available at: thttp://www.ema.europa eu/docs/en_GB/document_library/EPAR_-_Public_assessment_report/ human/002552/WC500166234.pdf. Accessed September 27, 2015.
16. European Medicines Agency. Deltyba (delamanid): Authorization Details. European Medicines Agency; 2014. Available from: http://www.ema. europa.eu/ema/index.jsp?curl=pages/medicines/human/medicines/ 002552/human_med_001699.jsp\&mid=WC0b01ac058001d124. Accessed September 27, 2015.

17. Otsuka Pharmaceutical Co., Ltd. After 40 years, a new drug for the treatment of tuberculosis in Japan [press release]. Japan: Otsuka Pharmaceutical Co, Ltd; 2014 [Jul 4]. Available from: http://www.otsuka. co.jp/en/company/release/2014/0704_02.html. Accessed September 27, 2015.

18. Otsuka Pharmaceutical Development and Commercialization, Inc. Pharmacokinetic and Safety Trial to Determine the Appropriate Dose for Pediatric Patients With Multidrug Resistant Tuberculosis. Available from: https://clinicaltrials.gov/ct2/show/NCT01856634?term=delam anid+pediatric\&rank=2. NLM identifier: NCT01856634. Accessed September 27, 2015.

19. Otsuka Pharmaceutical Development and Commercialization, Inc. A 6-Month Safety, Efficacy, and Pharmacokinetic Trial of Delamanid in Pediatric Patients With Multidrug Resistant Tuberculosis. Available from: http://clinicaltrials.gov/ct2/show/NCT01859923?term=delaman id\&rank=1. NLM identifier: NCT01859923. Accessed September 27, 2015.

20. European Medicines Agency. Summary of Opinion Initial Authorization. Deltyba (delamanid) Committee for Medicinal Products for Human Use (CHMP); 2013. EMA/CHMP/713909/2013. Available from: http:// www.ema.europa.eu/docs/en_GB/document_library/Summary_of_ opinion_-_Initial_authorisation/human/002552/WC500155458.pdf. Accessed September 27, 2015.

21. NHS England Specialised Services Clinical Reference Groups for Infectious Diseases and Respiratory Medicine. Clinical Commissioning Policy: Bedaquiline and Delamanid for Defined Patients with MDR-XDR-TB. NHS England; 2014. Available from: https://www.engage.england.nhs.uk/ consultation/specialised-services-consultation/user_uploads/bedaquilinedelamanid-policy-upd.pdf. Accessed September 27, 2015.

22. European Medicines Agency. Summary of product characteristics. Deltyba. Otsuka Novel Products GmbH. European Medicines Agency; 2014. Available from: http://www.ema.europa.eu/docs/en_GB/ document_library/EPAR_-_Product_Information/human/002552/ WC500166232.pdf. Accessed September 27, 2015.

23. Paccaly A, Petersen C, Patil S, et al. Absence of clinically relevant drug interaction between delamanid, a new drug for multidrug-resistant tuberculosis (MDR-TB) and tenofovir or lopinavir/ritonavir in healthy subjects. Poster presented at: 19th International AIDS Conference; July 22-27; 2012; Washington, DC.

24. Petersen C. Delamanid, a new drug for multi-drug resistant tuberculosis (MDR-TB), and efavirenz do not show clinically relevant drug interactions in healthy subjects. Poster abstract A-1255. Presented at: 52nd Interscience Conference on Antimicrobial Agents and Chemotherapy; September 9-12; 2012; San Francisco, CA.

25. Diacon AH, Dawson R, Hanekom M, et al. Early bactericidal activity of delamanid (OPC-67683) in smear-positive pulmonary tuberculosis patients. Int J Tuberc Lung Dis. 2011;15(7):949-954.

26. Gler MT, Skripconoka V, Sanchez-Garavito E, et al. Delamanid for multidrug-resistant pulmonary tuberculosis. N Engl J Med. 2012; 366(23):2151-2160.

27. Zhang Q, Liu Y, Tang S, Sha W, Xiao H. Clinical benefit of delamanid (OPC-67683) in the treatment of multidrug-resistant tuberculosis patients in China. Cell Biochem Biophys. 2013;67(3):957-963.

28. Skripconoka V, Danilovits M, Pehme L, et al. Delamanid improves outcomes and reduces mortality in multidrug-resistant tuberculosis. Eur Respir J. 2013;41(6):1393-1400.

29. Lewis JM, Sloan DJ. The role of delamanid in the treatment of drugresistant tuberculosis. Ther Clin Risk Manag. 2015;11:779-791.

30. Gupta R, Geiter LJ, Wells CD, Gao M, Cirule A, Xiao H. Delamanid for Extensively Drug-Resistant Tuberculosis. $N$ Engl $J$ Med. 2015;373(3):291-292. 
31. RESIST-TB [homepage on the Internet]. DR-TB Clinical Trial Progress Report. A Trial of the Safety, Tolerability, and Pharmacokinetics of Bedaquiline and Delamanid. Available from: http://www.resisttb. org/?page_id=1602. Accessed September 27, 2015.

32. Esposito S, D’Ambrosio L, Tadolini M, et al. ERS/WHO Tuberculosis Consilium assistance with extensively drug-resistant tuberculosis management in a child: case study of compassionate delamanid use. Eur Respir J. 2014;44(3):811-815.
33. Nachman S, Ahmed A, Amanullah F, et al. Towards early inclusion of children in tuberculosis drugs trials: a consensus statement. Lancet Infect Dis. 2015;15(6):711-720.

34. [No authors listed]. Access to medicines is a global struggle. The Pharmaceutical Journal. 2014;293:7830.

\section{Publish your work in this journal}

Infection and Drug Resistance is an international, peer-reviewed openaccess journal that focuses on the optimal treatment of infection (bacterial, fungal and viral) and the development and institution of preventive strategies to minimize the development and spread of resistance. The journal is specifically concerned with the epidemiology of antibiotic

\section{Dovepress}

resistance and the mechanisms of resistance development and diffusion in both hospitals and the community. The manuscript management system is completely online and includes a very quick and fair peerreview system, which is all easy to use. Visit http://www.dovepress.com/ testimonials.php to read real quotes from published authors.

Submit your manuscript here: http://www.dovepress.com/infection-and-drug-resistance-journal 